\title{
The Impact of Mental Aerobics Training on Older Adults
}

\section{Bert Hayslip Jr.', Kay Paggi ${ }^{2}$, and Daniela Caballero ${ }^{3}$}

\begin{abstract}
Mental Aerobics (MA) is a cognitively oriented intervention designed to improve older adults' perceptions of their cognitive ability as well as positively impact their performance. Forty-seven community-residing older adults $(M$ age $=67.39, S D=5.75)$ were randomly assigned to either a treatment $(n=24)$ or a waiting list control $(n=23)$ group. Participants in both treatment and control conditions completed measures of depression, self-rated health, cognitive functioning, and cognitive self-efficacy. Findings support the conclusion that MA can be an effective intervention in improving older adults' affectivity, everyday task self-efficacy, and self-rated health. The impact of MA on measures of generalized fluid ability (Gf) and generalized crystallized ability $(G c)$ skills was moderated by level of education. The subsequent provision of MA training to controls minimized the performance differences between the treatment and control conditions, as well as the moderating effect of level of education. These findings suggest that MA can be effectively used to enhance older adults' views of their skills, critical to efforts to maintain cognitive functioning into later life, although some older persons may benefit to a greater extent than others.
\end{abstract}

Manuscript received: July 16, 20I4; final revision received: January 2, 2015; accepted: February 21, 2015.

'University of North Texas, Murrells Inlet, SC, USA

2Private Practice, Dallas, TX, USA

${ }^{3}$ University of North Texas, Denton, USA

\section{Corresponding Author:}

Bert Hayslip Jr., University of North Texas, 82I Sail Ln \#I0I, Murrells Inlet, SC 29576, USA.

Email: BertHayslip@my.unt.edu 


\section{Keywords}

mental aerobics, older adults, lifelong learning

Cognitive engagement and social activity are each in isolation minimal in delaying the onset of Alzheimer's disease (AD), though physical activity and cognitive interventions may each have a small protective factor in delaying cognitive decline (Williams, Plassman, Burke, Holsinger, \& Benjamin, 2010). Importantly, Park, Gutchess, Meade, and Stine-Morrow (2007) cite several studies suggesting that both cognitive and social engagement can be protective and further suggest that the success of such interventions is associated with the formation of new neural pathways. Key to such claims is the notion of productive engagement, which involves the learning of new skills (Hertzog, Kramer, Wilson, \& Lindenberger, 2009; Park et al., 2007; StineMorrow, 2007; Stine-Morrow, Parisi, Morrow, Greene, \& Park, 2007). Likewise, the reported success of the Experience Corps, where older adults serve as mentors in the public schools with benefits for themselves and the children with whom they work (see Fried et al., 2003), as well as the current popularity of "brain exercises" (see, for example, www.luminosity.com) reflect the interest in efforts promoting cognitive engagement in later life.

Indeed, unchallenged assumptions about declines in cognition could affect an older person's self-esteem, further exacerbating potential depression and isolation, contributing to further losses in cognitive functioning. That this may be the case is evidenced by the work of Hess, Auman, Colcombe, and Rahhal (2003) who found that when older adults are exposed to negative stereotypes about memory and aging they perform more poorly on memory recall tasks than do their counterparts who are not exposed to such negative stereotypes. This poor performance and the subsequent interpretation of it lead to decreased cognitive self-efficacy. Indeed, older adults who believe that their cognitive skills are deficient (see Hayslip \& Cooper, 2012; Schaie, Willis, \& O'Hanlon, 1994) may avoid activities that require cognitive effort (Paggi \& Hayslip, 1999). By avoiding such activities, they lose opportunities to contradict their belief in their own perceived cognitive decline.

Mental Aerobics (MA), developed by the second author in 1993, incorporates many of these elements (productive engagement, cognitive stimulation, social engagement). MA (see Paggi \& Hayslip, 1999) is partly based spontaneous drills used for creative thinking practice (see Parisi, Greene, Morrow, \& Stine-Morrow, 2007). MA is designed to enhance adults' cognitive selfesteem and improve everyday (cognitive) functioning, wherein in addition to spontaneous creativity drills, MA also uses math problems, spatial puzzles, 
verbal exercises, and other mental games to stimulate thinking. Importantly, MA is interactive; it is a challenging, yet entertaining experience for older adults in the company of age peers who are also motivated to use and improve their mental skills (Paggi \& Hayslip, 1999). It therefore is both social and cognitive in nature, combining factors which have been noted as potentially protective in minimizing cognitive decline in later life (Stine-Morrow \& Basak, 2011; Williams et al., 2010).

MA's dual emphasis upon social and cognitive engagement is also consistent with the impact of noncognitive factors on cognitive functioning in later life (Hayslip \& Maiden, 2005). Moreover, the interpersonal nature of MA is reflected in the attention paid to the interpersonal and emotional influences on later life cognition (see Hayslip, 1989a, 1989b; Williams et al., 2010). This is significant in that there are relationships between engagement in cognitively stimulating social and mental activities and what has been termed cognitive vitality (see, for example, Hultsch, Hertzog, Small, \& Dixon, 1999; Stine-Morrow \& Basak, 2011).

Reflecting the fact that the efficacy of cognitive (memory) training may covary with its multimodal nature (Stine-Morrow \& Basak, 2011), MA is multimodal, stressing (a) the creative use of one's cognitive skills to solve challenging problems in a supportive group atmosphere, (b) the active involvement of each older person, (c) solutions to problems that are both individually and collectively reached in the presence of supportive and encouraging group leader, and most importantly, and (d) the process of contributing to the solution of problems (e.g., sharing of ideas, interacting with others, getting feedback about the solution to a problem that has been proposed, being prompted by others for guesses).

In this light, making possible interactions that are personally meaningful is a fundamental tenet of MA (Paggi \& Hayslip, 1999). Thus, while MA is would likely be considered a cognitively oriented intervention, it also requires the active social engagement of older adults. Thus, it may have secondary and/or indirect benefits in changing the social-emotional-interpersonal context in which older persons function, key to the effective use of their mental skills. In addition, in this respect, the content of MA problems is quite diverse (Paggi \& Hayslip, 1999) and can be adapted to the skills and interests of older persons in different settings.

Although anecdotal evidence (Paggi \& Hayslip, 1999) suggests MA to be effective with community-residing older adults, and published work suggests that it may be effective with memory-impaired older adults (Hayslip, Paggi, Poole, \& Ward Pinson, 2009), empirical evidence for its short-term and longterm effectiveness with community-residing older persons has yet to be published. In this light, Stine-Morrow and her colleagues (Stine-Morrow et al., 
2007; Stine-Morrow, Parisi, Park, \& Morrow, 2008) have provided evidence suggesting that a variation on the Odyssey of the Mind (the Senior Odyssey) was impactful in improving the cognitive performance of older participants, having pseudorandomly assigned older adults to an experimental and control condition, based on pretest and 6- to 8-month posttest follow-up data. However, the program was not effective in changing dispositional constructs, that is, mindfulness, self-efficacy, thought to be key to continued intellectual engagement in later life (Stine-Morrow et al., 2008).

The present study builds on not only the work of Paggi and Hayslip (1999) in evaluating the short-term and long-term efficacy of MA but also that of Stine-Morrow and her colleagues, who for practical reasons, were not able to randomly assign all participants to a treatment or control condition, and whose Senior Odyssey research did not involve a long-term follow-up. In addition, the control group utilized by Stine-Morrow and her colleagues appeared to be a no-contact control, versus in the present study, where participants were randomly assigned to a treatment or a waiting list control condition, to in part, control for expectancy effects (see below). Indeed, Stine-Morrow and Basak (2011) raise this issue in light of the applicability of the standard against which treatment/intervention effects with older adults might be judged.

In the context of evaluating the short-term and long-term efficacy of MA training, we hypothesized that MA would yield superior short-term cognitive and affective functioning as well as more efficacious perceptions of one's everyday cognitive skills among such persons, relative to a waiting list control condition, and that such effects would be maintained over time. Moreover, we expected that subsequent MA training provided to waiting list control participants would yield similar gains in perceptions of everyday skills, cognitive and affective functioning. We also explored level of education as a potential moderator of MA training effects (see Stine-Morrow \& Basak, 2011; Stine-Morrow et al., 2008).

\section{Method}

\section{Participants}

This project was funded by a small grant to the first author from University of North Texas (UNT), and was approved by the UNT Institutional Review Board (IRB) for the Protection of Human Subjects in Research, wherein each participant first completed an IRB approved Informed Consent Form prior to any data collection. Fifty-four community-residing older persons who expressed an interest in "developing their abilities to the fullest" were 
recruited from the community at large through presentations to older adult organizations such as American Association of Retired Persons (AARP) and National Association of Retired Federal Employees (NARFE) as well as newspaper announcements of the project. The sample consisted of 41 women and 12 men (missing data $n=1$ for gender) between the ages of 59 and 84 ( $M=$ $69.15, S D=7.06$ ). All but one participant identified himself or herself as Caucasian. The 41 participants who disclosed their income averaged US $\$ 30,000$ to US $\$ 40,000$ annually. The average participant reported completing some college and half of all participants had obtained a college degree or beyond. On average, participants rated their health as "good" relative to others of their age (where $1=$ poor and $5=$ excellent, $M=3.91, S D=.88$ ). They reported being moderately concerned about their own aging, where $1=$ not concerned at all and $5=$ being very upset, $M=3.41, S D=1.15$. Table 1 presents the demographic characteristics of the sample for the MA and waiting list control groups (see below), based on complete data for the sample as a whole for all demographic variables.

\section{Measures}

Participants completed a short battery on three separate occasions (pretest, 1-week posttest, 1-year posttest) consisting of self-report scales assessing intellectual self-efficacy (Personality in Intellectual Aging Contexts Inventory, Lachman, Baltes, Nesselroade, \& Willis, 1982), depression (Geriatric Depression Scale-Short Form, Sheikh \& Yesavage, 1986), and a measure of anxiety about aging constructed for purposes of the present study (35 items reflecting concerns about the cognitive, physical, and interpersonal dimensions of aging, for example, changes in one's appearance, memory loss, loneliness, daily functional ability, losses in vision and hearing, $\alpha=.95$ ). They also completed measures of everyday cognitive failures (Broadbent, Cooper, Fitzgerald, \& Parkes, 1982), a 20-item quality of life measure SF (Short Form Health Status)-36, reflecting physical functioning, role functioning, mental health, physical and role functioning; see Ware, Snow, Kosinski, \& Gandek,1993). In addition, persons completed measures indexing selfrated self-efficacy, that is, "how good are you at ...?" and intellectual health and vitality, that is, "how important is . . to you in helping to maintain your intellectual health and vitality/being able to use your mind in an active way?" as each applied to 10 ecological (e.g., household maintenance, shopping, reading maps and schedules, understanding directions) and to 10 nonecological (arithmetic, vocabulary, memory for names, place and digits, anagrams) problems (Hayslip, Servaty, \& Ward, 1996; Hayslip, Servaty, Ward, \& Blackburn, 1995; Hayslip \& Thomas, 1999). In addition, persons completed 
Table I. Demographic Characteristics for the MA and Waiting List Control Groups.

\begin{tabular}{lc}
\hline MA & Waiting list control \\
\hline Age & $70.82(7.38)$ \\
$67.28(6.30)$ & $93 \%$ Caucasian \\
Ethnicity & $3 \%$ Hispanic $(n=I)$ \\
$100 \%$ Caucasian & $3 \%$ Missing $(n=1)$ \\
& \\
Gender & $M=7, F=16$ \\
$M=5, F=19$ & \\
Income & $5.17(1.82)$ \\
$5.67(I .64)$ & $14.66(3.94)$ \\
Education in years & \\
I $4.44(5.04)$ & $52 \%$ married \\
Marital status & \\
$76 \%$ married & $3 \%$ divorced $(n=1)$ \\
I $2 \%$ divorced & $14 \%$ single \\
I $2 \%$ widowed & $28 \%$ widowed \\
& $3 \%$ missing $(n=1)$ \\
\hline
\end{tabular}

Note. Annual income was coded along a continuum of seven increments, ranging from less than US\$I5K to more than US\$50K, with values of 5 equally between US $\$ 30 \mathrm{~K}$ and US\$40K annually. One individual failed to provide data regarding gender. $M A=$ Mental Aerobics.

measures of fluid (Gf-letter series) and crystallized (Gc-vocabulary) ability (Hayslip, 1989a; Hayslip \& Sterns, 1979). Alpha coefficients exceeded .80 for nearly all measures, wherein the lowest in this respect was .75 for the depression measure in the present sample.

\section{Procedure}

Participants were randomly assigned to a 6-session 1-hr MA training program (complete data for $n=24,5$ men, 19 women), which met once a week, or to a waiting list control condition (complete data $n=23,7$ men, 16 women) where they were told that the current training group was filled but they would be included in the next scheduled group. For such persons, at the conclusion of the 1-week posttest, this training did indeed occur; this constitutes a potential replication of any earlier training effects established via an earlier shortterm comparison of the pretest-posttest scores of the experimental and 
control participants. Thus, based on their prior waiting list control status (controlling for expectancy effects), control group participants were offered the opportunity to receive MA training identical to that of the original treatment group.

MA training and waiting list control participants were similarly aged and educated, similar regarding gender, and scored similarly (as a set and on an individual basis) on measures of self-reported health, self-efficacy, letter series, vocabulary, everyday cognitive failures, depression, and both selfrated overall intellectual vitality and competence as per a MANOVA and a series of ANOVAs ( $p>.05$ in each case).

\section{Description of the MA Program}

In the MA program for both the experimental and later so for the waiting list control participants, initial sessions included several different types of activities including spontaneous drills, math, logic, and verbal problems. Examples of such activities include the following: rebuses - a picture of the word "sand" written within a box, where this is a rebus for "sandbox"; naming a grandbaby whose initials are WWW where the parents" last name is "White"; naming body parts beginning with the letter " $\mathrm{A}$ " through the alphabet; using names of candy to fit a described phrase - stars in the sky-Milky Way; deriving one's birth date by performing a series of arithmetic operations beginning with the month one was born; verbal doublets - making the word "Bread" in the word "Flour" by changing only one letter at a time, leaving the remaining letters in their respective positions; word equations $-26=\mathrm{L}$ of the A is 26 letters of the alphabet; oxymorons_- "jumbo shrimp," "original copy." In addition, activities designed to help participants get to know one another (e.g., learning one another's names using a mnemonic) defined each session. As sessions progressed, the cognitively engaging activities became more complex but still covered math, logic, and verbal-type problems.

It is important to note that in each session, effortful and collaboratively creative problem solving was stressed, wherein the emphasis upon solving the problem per se, that is, providing a "correct" answer, was minimized. Indeed, "cheating," that is, encouragement and hint-dropping from the group leader and/or group members, was encouraged to help contribute to the cooperative atmosphere in each session. Problems were presented as interesting, unique, and challenging in nature.

One week prior to training and 1 week after training had been completed, all participants completed the above battery of measures, with the MA and waiting list control participants completing the battery at similar times. Approximately 1 year after the immediate posttest surveys completed, all 
individuals were approached for a follow-up assessment. Seventy-five percent of the original participants (those with compete data at the 1-week posttest) completed the battery of measures 1 -year later; individuals were equally likely (experimental, $n=19,74 \%$; waiting list control, $n=20,76 \%$ ) to complete the 1-year posttest.

\section{Results}

\section{Analytic Plan}

Utilizing SPSS Version 22, a series of ANCOVAs were conducted with the 1 -week posttest measure as the dependent variable, group membership (MA training or waiting list control) and potential moderating variables (i.e., education viewed either as predispositional or protective factors in the maintenance of one's skills; see Stine-Morrow \& Basak, 2011; Stine-Morrow et al., 2008) as between-subject factors, and the pretest as the covariate.

\section{Short-Term Findings: Main Effects for MA Training}

Preliminary analyses of T1 (pretest) and T2 (immediate posttest) data pertinent to the major cognitive and affective variables of interest here, for example, measures of crystallized and fluid ability, depression, intellectual self-efficacy, indicated no main effects for gender or interactions between gender and group membership. Consequently data were pooled across gender in the analyses reported below.

The use of the respective pretest as a covariate addressed the likelihood of practice and/or sensitization effects on measures of cognition, cognitive selfefficacy, and dispositional/protective factors, for example, health and affective function, as influences on the data over time. Findings based on these ANOVAs (see Table 2) suggested that MA participants' posttest depression scores were lower than those of the control group, $F(1,42)=5.42, p<.03$, $\eta_{\mathrm{p}}^{2}=.11(\operatorname{Adj} M=13.57$ vs. Adj $M=14.41, d=70)$. The Cohen's $d$ effect size is at least moderate in nature.

Although not statistically significant, these analyses also suggested that MA participants tended to place more importance on their ability to handle finances in maintaining their intellectual health and vitality (where lower scores indexed more vitality) than did the waiting list group, $F(1,44)=3.10$, $p<.08, \eta_{\mathrm{p}}^{2}=.07$ (Adj $M=11.04$ vs. Adj $M=12.72, d=.54$ ). MA participants also tended to rate their health as better (i.e., they reported fewer health limitations), relative to waiting list controls, $F(1,43)=3.03, p<.09, \eta_{\mathrm{p}}^{2}=.07$ (Adj $M=14.88$ vs. Adj $M=14.01, d=.53$; see Table 2). In both of these 


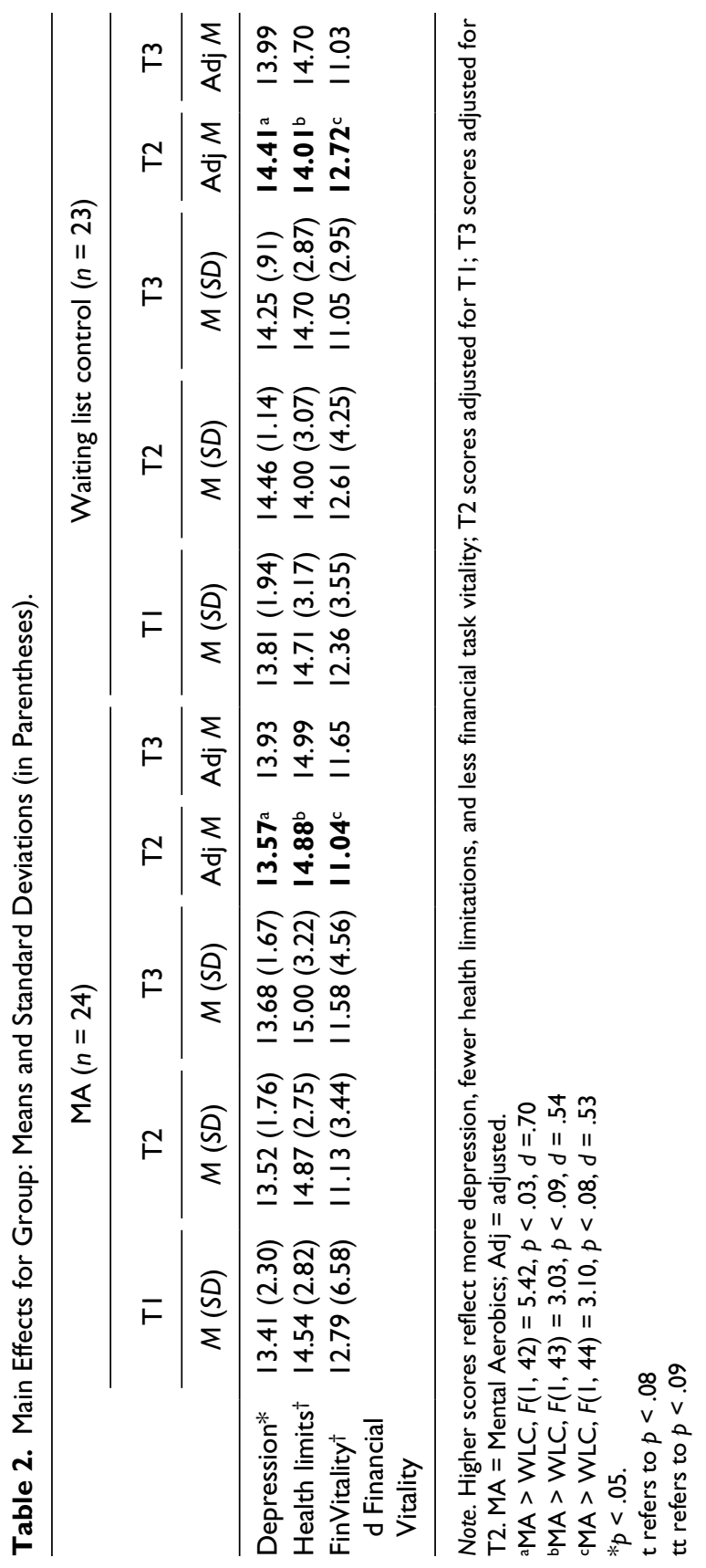


cases, though statistically nonsignificant $(p<.09)$, the Cohen's $d$ effect sizes each approach moderate in nature and exceed the standard for a weak effect (.20; see Cohen, 1988).

\section{Level of Education as a Moderator of Training Effects}

Having dichotomized the sample into those with and without a college degree ( $50 \%$ of the sample reported having attained each), the level of education by treatment interaction impacted both vocabulary, $F(1,48)=4.35, p<.05, \eta_{\mathrm{p}}^{2}=$ .08 , and letter series, $F(1,47)=5.49, p<.02, \eta_{\mathrm{p}}^{2}=.10$, performance (see Table 3). Among MA participants, those with more education scored more highly on letter series (Adj $M=10.38$ vs. Adj $M=8.62, d=.66$ ) and vocabulary (Adj $M=12.45 \mathrm{vs.} \mathrm{Adj} M=10.53, d=.58$ ), while among controls, those with less education scored more highly on letter series (Adj $M=9.48$ vs. Adj $M=7.67, d=.69$ ) and vocabulary (Adj $M=13.43$ vs. Adj $M=12.92, d=.49$ ), these Cohen's $d$ effect sizes are clearly moderate in nature.

This interaction was, strictly speaking, statistically nonsignificant for the importance of solving everyday problems attached to maintaining one's intellectual health and vitality, $F(1,43)=3.05, p<.08, \eta_{\mathrm{p}}^{2}=.07$, wherein in MA participants, scores did not vary between persons who were in varying degrees, highly educated (Adj $M=16.06$ vs. Adj $M=15.70, d=.20$ ), while among waiting list controls, those who were less highly educated tended to place more importance on solving everyday problems as a source of intellectual vitality (where lower scores indexed greater vitality) (Adj $M=17.55$ vs. Adj $M=14.15, d=.73$; see Table 3 ). In this latter comparison, the Cohen's $d$ effect size was moderate.

\section{Main Effects for Level of Education}

Those who were more highly educated placed less importance (where higher scores indexed less vitality) on being able to solve everyday problems in maintaining their intellectual health and vitality, $F(1,43)=4.76, p<.03, \eta_{\mathrm{p}}^{2}=.10$ (Adj $M=14.92$ vs. Adj $M=16.87, d=.66$ ), being able to competently carry out housekeeping chores, $F(1,44)=3.95, p<.05, \eta_{\mathrm{p}}^{2}=.08(\operatorname{Adj} M=19.82 \mathrm{vs}$. Adj $M=22.10, d=.71)$, and being able to remember important names and dates, $F(1,43)=4.52, p<.04, \eta_{\mathrm{p}}^{2}=.09($ Adj $M=19.71$ vs. Adj $M=22.16, d=.62)$. Such persons were, however, less anxious about aging, $F(1,35)=4.22, p<.05$, $\eta_{\mathrm{p}}^{2}=.11$ (Adj $M=94.86$ vs. Adj $M=85.33, d=.65$; see Table 4).

Persons who were more highly educated also tended to attach less overall importance to maintaining their intellectual health and vitality (where higher scores indexed less vitality), $F(1,42)=3.14, p<.08, \eta_{\mathrm{p}}^{2}=.07$ (Adj $M=78.42$ 


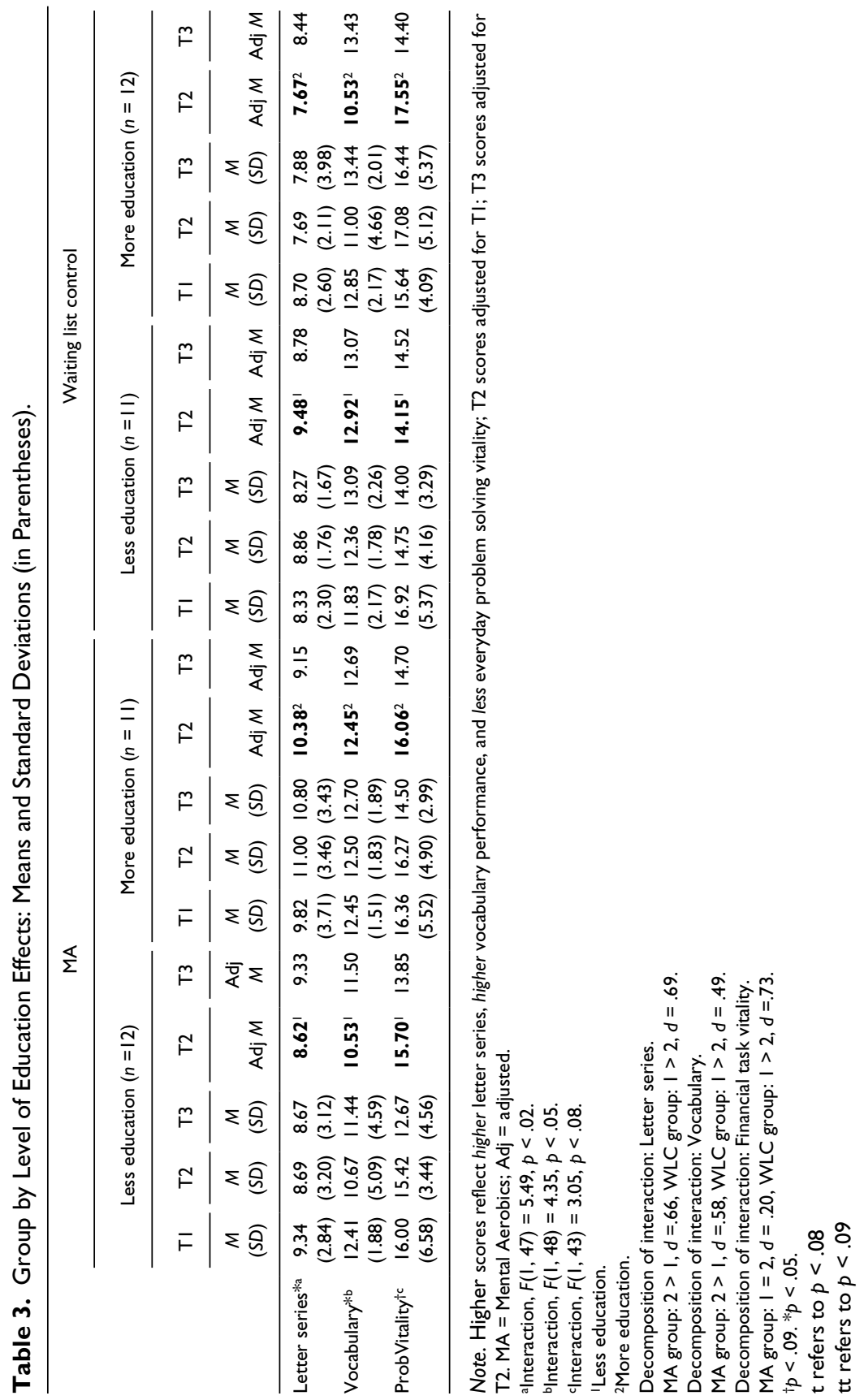




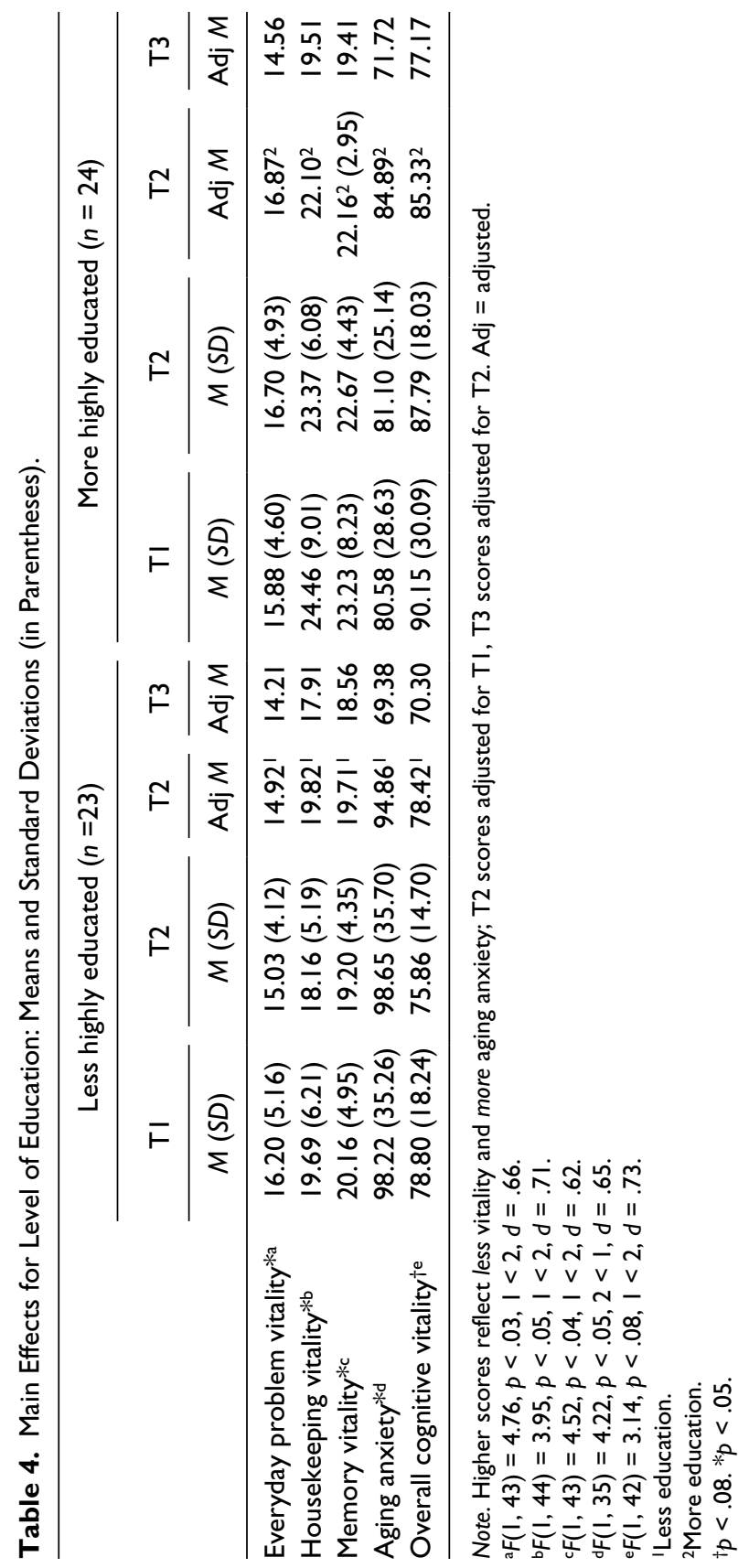


vs. Adj $M=85.83, d=.73$ ), relative to those who were less highly educated, though this effect is, strictly speaking, statistically nonsignificant. Yet, its Cohen's $d$ exceeds being moderate in nature.

\section{Long-Term Follow-Up Effects of MA Training and Replication of Short-Term MA Training Effects}

Given the lower depression scores among MA participants at T2 (adjusting for such scores at T1), a paired $t$ test of the T2 and T3 (adjusting for T2) depression scores particular to the training group suggested that the above gains in affective status were maintained over the 1-year time period $\left(t_{18}=\right.$ $-.51, p>.05)$. This was confirmed via the high correlation between $\mathrm{T} 2$ and T3 scores in MA participants $(r=.67, p<.01)$ and a nonsignificant $(p>.05)$ effect of adjusting T3 depression scores for the effects of T2 depression.

Subsequent MA training which had earlier been provided to control participants constituted a replication of MA training effects, wherein in this case, 1-year data were the dependent variable and the 1-week posttest scores were the covariate. This training provided to the waiting list control group minimized $(p>.05)$ earlier observed differences in depression which had favored the treatment group as well as formerly statistically significant interactions between treatment and level of education with regard to letter series and vocabulary performance as well as everyday problem solving pertinent to one's intellectual health and vitality $(p>.10)$. At T3, there were no longer main effects for education.

A series of group and/or group by education repeated-measures ANOVAs and ANCOVAs (pretest as covariate) confirmed that the above group effects as well as the above group by education interactions involving the 1-year data were not artifacts of the MA groups' scores on letter series, vocabulary, depression, financial task, intellectual health and vitality, and self-rated physical health declining from the immediate posttest to follow-up, regardless of whether or not controls for the pretest were utilized; supporting this finding was the fact that there was no significant group by occasion interactions for the 1-week and 1-year posttest data.

\section{Discussion}

\section{The Impact of MA Training}

The purpose of this study was to empirically evaluate the impact of MA training on older adults. As either the disuse of one's skills or a loss of confidence in the consequences of the use of such skills may either be mistaken for or 
contribute to cognitive decline, the potential impact of the MA program, designed to positively effect both cognitive performance and cognitive selfefficacy, is of particular value.

Findings here suggested that the effects of MA training per se (as well as its interaction with level of education, see below) transcend cognitive performance per se, and are better thought as being broad spectrum in nature (see Stine-Morrow \& Basak, 2011). They moreover suggest that simply believing that one might be the beneficiary of training, that is, expectancy effects, is not a viable explanation for these findings.

In terms of its value in impacting cognitive performance, while MA participants' finance-related vitality scores only tended to be higher than those of waiting list controls, findings here clearly support the role of MA in enhancing the positive role of those predispositional and/or protective factors, that is, less depression, in the maintenance of one's skills (see Stine-Morrow \& Basak, 2011), wherein MA participants reported less depression and tended to report better physical health relative to waiting list control group participants (see Table 2). While MA participants' depression scores being lower could contribute to their reports of better health or intellectual vitality, future work might explore these factors as contributors to the benefits of MA training for older persons. Indeed, as depressed older persons are often sensitized to failure (see Blazer, 2002), the lessening of depressive symptoms via MA may predispose such persons to approach difficult and/or challenging tasks in a more self-confident and proactive manner.

\section{The Moderating Role of Education on MA Program Efficacy}

As more education may contribute to the maintenance of cognitive functioning over time (Albert et al., 1995; Williams et al., 2010), it is not surprising that the effects of MA were moderated by level of education. The statistically significant group by level of education interactions here suggested that depending upon the moderating effects of level of education, MA training versus waiting list control participants were each positively affected regarding both fluid and crystallized ability, addressing the question of whether all older persons necessarily benefit from a given intervention to an equal extent (see Hayslip, 1989a, 1989b; Kooken \& Hayslip, 1984; Pashler, McDaniel, Roher, \& Bjork, 2009; Stine-Morrow \& Basak, 2011).

Indeed, we found that those who were more highly educated benefited from MA training to a greater extent in terms of letter series (Gf) and vocabulary (Gc) performance (see Table 3). This might be explained via the MA program's reinforcing their beliefs about the adequacy of their skills, borne of having been more frequently intellectually challenged in the past and 
consequently being more attuned to the cognitive benefits of being more highly educated.

Thus, more highly educated older persons may have benefited from MA training to a greater extent in that it enabled them to deal with the sensitivity to the loss of their skills, influenced by how they expect themselves to be able to perform, having been challenged more frequently in the past, being more highly educated. For this reason, they may put forth more effort to increase their engagement in the cognitively challenging activities characteristic of the MA program. As such persons may be more attuned to how they should be performing, their efforts in the MA program may be driven by previously established expectations regarding their problem-solving skills, resulting in greater performance $(\mathrm{Gf} / \mathrm{Gc})$ benefits for them. For more highly educated older persons, "use or lose it" may indeed an operative motivational factor driving the benefits of MA training to them, relative to persons in the MA training group who are less highly educated.

The pattern of differential MA group performance on letter series and vocabulary by level of education might also be attributed to the relatively proactive nature of the group problem-solving process characterizing MA training, where those who are more educated may take a more active role in generating solutions to the problems presented to them. In contrast, persons who were less highly educated and somewhat passive in this respect perhaps took fewer risks in generating solutions during the MA sessions that might be seen as incorrect or at least felt that it was less necessary for them to provide an answer to a given problem to be solved, given that others were more likely to do so.

The above discussion suggests that for some older persons (i.e., those who are more highly educated), the effects of MA observed here can be understood in terms of the benefits of a cognitively engaged lifestyle - being productively and effortfully engaged. To an extent, this may protect persons against future declines in cognitive functioning and promote future efforts suggesting that the more highly educated are more cognitively engaged (Andel et al., 2005; Boron, Willis, \& Schaie, 2007; Schooler, Mulatu, \& Oates, 1999; Snowdon, 2001; Williams et al., 2010). Importantly in this respect, the lessened depression and, to an extent, both better self-reported health and greater financial task vitality associated with MA training may also be protective, leading to further cognitive engagement.

Somewhat surprisingly, among waiting list controls, those who did not have college degrees scored more highly on both vocabulary and letter series tasks, as well as (to a lesser extent) rated more highly the personal importance (vitality) of being able to solve everyday problems, compared to persons who were more highly educated in the waiting list control 
condition. At the minimum, this contradicts the common assumption that being more highly educated necessarily advantages persons cognitively, especially with regard to crystallized ability (see Horn \& Cattell, 1966, 1967; Horn \& Hofer, 1992).

This unusual pattern of findings leads to some interesting, albeit speculative, potential explanations regarding the differential role that level of education may play in the absence of MA training in affecting letter series and vocabulary performance, and to a somewhat lesser extent, everyday problemsolving vitality.

It may be that more highly educated people in the waiting list control group may be more sensitive to potential failures in solving challenging problems, that is, letter series and vocabulary tasks, relative to such persons who are less highly educated, in that the former may be more highly sensitized to how they should be performing, cognitively speaking (see above), and consequently make greater efforts to solve letter series and vocabulary tasks, which in this case, undermined their performance. This interpretation is in part buttressed by the fact that older persons scoring more highly on measures of Gc are more anxious about losing their cognitive skills (Hayslip, 1988), and the fact that anxiety reduction training provided to older persons can enhance their Gf performance (Hayslip, 1989a, 1989b). It is also consistent with the above discussed facilitative effect of MA on the Gf/Gc performance of more highly educated older persons. It would follow that less highly educated persons in the waiting list control group might be less likely to be threatened by demanding tasks, not having developed expectations regarding how they should be performing. This comparative lack of concern about the adequacy of their skills could help to facilitate better cognitive (Gf/Gc) performance, to the extent that they attach less importance to performing poorly on such tasks. Likewise, having taken fewer risks in generating solutions to MA-type problems, they were less attuned to failing difficult and/or challenging tasks such as letter series or vocabulary.

In light of the above, albeit speculative, discussion of those factors potentially explaining the differential performance in the MA versus waiting list control groups regarding letter series and vocabulary performance, future research might explore in depth what it is about being more or less highly educated that affects MA training outcomes, for example, do such persons have different expectations regarding their cognitive performance? Likewise, more work is needed that addresses the interpersonal dynamics of problem solving in a group setting. Future work exploring the dynamics of persons' reactions to challenging tasks as influences on the outcomes of interventions such as MA training might also be promising in this respect. 


\section{The Effects of Level of Education}

Despite the above findings regarding the interaction of education and MA training, the main effect found here for level of education may reinforce being more highly educated as a protective factor regarding late life cognitive functioning in an interesting manner, though perhaps in some unexpected ways. In that more highly educated persons (surprisingly) reported less everyday task vitality, less housekeeping task and memory vitality, and less overall intellectual health and vitality (see Albert et al., 1995; Stine-Morrow et al., 2008), but nevertheless reported being less anxious about aging might suggest that being able to perform such everyday tasks added little to how they already felt about their intellectual vitality, borne of having already experienced greater intellectual challenge via more education, especially in light of their fewer reported concerns about growing older. In contrast, less highly educated persons were more anxious about growing older consequently ascribed more cognitive importance, that is, vitality, to being able to perform well in a variety of everyday domains (see Table 4).

It thus appears that for differing sets of reasons, less highly educated and more highly educated persons not only differentially benefit from MA training - a group-based psychosocial cognitive intervention — but also that they need to be differentially understood metacognitively in light of the value they may place on everyday experience. That is, more highly educated older persons may be more cognitively proactive and driven by perceived discrepancies between how they should be performing and their actual performance, but yet see little to be gained by everyday cognitive engagement as a defense against loss, in that they are less anxious about aging. In contrast, less highly educated persons may be less threatened by the loss of their skills, leading to better cognitive performance (see above). Yet, they perceive more to be gained by greater everyday cognitive engagement, driven by greater fears about growing older. Thus, each subset of persons, motivationally speaking, may be more likely to remain cognitively engaged and/or initiate efforts to improve or maintain their skills.

It is to be noted that level of education did not differentiate persons regarding Gf and Gc performance. This is likely an artifact of the positively biased nature of the sample regarding education, where $78 \%$ of persons had at least 1 year of college. Moreover, as noted above, provision of training to waiting list controls eliminated $(p>.05)$ the earlier main effects for level of education.

\section{The Replication of MA Training Effects}

Importantly, when waiting list controls were provided MA training after the 1-week posttest, 1-year findings (controlling for 1-week posttest scores) 
suggested that such training minimized the earlier found treatment/control differences in depression (clearly favoring MA participants), everyday (finances) task vitality and physical health (less robust, but still favoring MA participants), as well as eliminating the earlier found $(p<.05)$ treatment by level of education interactions for Gf and Gc and those which were somewhat less robust (everyday task vitality). This minimization of MA training/waiting list control differences was not an artifact of declines over time in the MA training group. Provision of training to waiting list controls also eliminated the main effects for level of education found at T2 (see above).

Overall, the original MA effects findings and those pertinent to the subsequent MA training provided to waiting list control participants suggest that the provision of MA training may mimic the effects of the continued use of one's skills and/or the positive effects of either level of education and/or dynamic interactions with others on both depression, physical health, cognitive vitality, or cognitive functioning per se (see Williams et al., 2010).

Despite a few construct-specific effects associated with MA training and/ or its interaction with education (such as an increase in the importance placed on handling finances), it seems that the (differential) effects of this intervention are more broad than specific, especially as they relate to the impact of the program as it interacted with level of education on both crystallized and fluid ability as well as the main effect for MA training on depression, and to an extent on cognitive vitality and physical health. It is our opinion that such changes were brought about by older adults' actively participating in the process of creatively and proactively generating solutions to challenging tasks and finding they were still quite capable of doing so. This interpretation is reinforced by the fact that MA training benefits were replicated when the waiting list control group received subsequent MA training.

It is also likely that the social stimulation and interactions with age peers who shared a desire to improve their mental skills, inherent in the program's design and structure, contributed to feelings of competence and positive affect. That participants placed more importance on handling their finances after participating in the program could be interpreted as older adults now emphasize the importance of being able to competently engage in a cognitively challenging activity because they indeed feel they are now more capable of performing such a task. In this respect, it may be that those older adults (i.e., those in the waiting list control group) who do not feel competent at performing well in challenging tasks minimize the importance of doing so to defend themselves against feelings of incompetence and failure. That they would report more depressive symptoms and to an extent, rate their health as poorer suggests that they may pay an affective and functional price in doing so. Moreover, upward social comparisons to others who are perceived as 
more competent as well as the stereotype threat generated by such comparisons might explain the differential MA versus waiting list control group impact on persons varying by level of education (see Hess et al., 2003).

\section{Limitations and Future Directions}

Although the results of this pilot study are encouraging, building upon earlier work by the first and second authors (Paggi \& Hayslip, 1999), and in part replicate and extend those of Stine-Morrow and her colleagues with the Senior Odyssey program, there are limitations here that should be acknowledged. Although we controlled for expectations of change via the implementation of a waiting list control group, it is possible that the mere interaction between participants in any context could produce patterns similar to those found here. However, this seems unlikely, especially with regard to the program's impact on cognitive functioning (as it interacted with level of education), wherein, generally speaking, the literature supports the roles of heuristics training, anxiety reduction, and/or practice in enhancing Gf/Gc performance (see, for example, Baltes \& Willis, 1982; Hayslip, 1989a, 1989b; Hayslip \& Maiden, 2005). Thus, in the context of an environment geared to collaborative problem solving, that is, the MA program, it seems unlikely that an interaction-neutral condition would produce measurable and reliable gains parallel to those found here. However, in the strictest sense, the impact (if any) of social interaction per se on affectivity, cognitive self-efficacy, or cognitive functioning cannot be evaluated here.

The sample here was not diverse in terms of ethnicity, income, health, and, to a certain extent, education (many participants had attended some college). Thus, these findings may not generalize to those who are less affluent, less highly educated, or in poorer health. Likewise, the breadth of the effects of MA and/or its interaction with education did not generalize to measures of cognitive self-efficacy (Lachman et al., 1982), quality of life, overall assessments of how good people were at a variety of tasks, or the overall importance they assigned to doing well on tasks that are key to their intellectual health and vitality. Likewise, findings regarding cognitive functioning (e.g., $\mathrm{Gf} / \mathrm{Gc}$ ) may not generalize to other measures of cognitive functioning (e.g., memory, divergent thinking, executive functioning), and we do not know whether being exposed to MA training predisposed persons to continue their efforts to improve their skills after the program's end, for example, enrolling in a college class, engaging oneself via brain stimulating activity, that is, Luminosity. This, of course, applies both to the original MA training participants and to those in the waiting list control who were subsequently provided with MA training. 
In this respect, the power of more sophisticated multivariate techniques to explore treatment and/or moderation effects was compromised here given the relatively small sample sizes; this should be taken into account when interpreting the present findings. A similar lack of power might explain the pattern for certain variables whose effects were strictly speaking, not statistically significant, that is, perceptions of one's health and views about everyday cognitive skills. However, in almost all of these cases, effect sizes, as assessed via partial eta squared and/or Cohen's $d$ were at least moderate in nature or approached being moderate - that is, they exceeded the standard for a small effect size (.20); see, for example, Cohen (1988). Thus, we chose to discuss findings that were, strictly speaking, statistically nonsignificant, as their effect sizes either approached or exceeded being moderate in nature. Consequently, we saw them as meaningful, despite the likely lack of power via the small sample here in influencing the odds of rejecting the null hypothesis of no main effects or interactions. This being said, there is still a need for samples that are larger than those here to evaluate the impact of MA training on older persons, as well as its differential benefits for some older persons and not others.

In addition, this sample may be unique in that participants were likely already concerned about losing their skills and consequently motivated to improve. Yet, MA training's effects (e.g., depression) were superior to those of waiting list controls, suggesting that expectations for change per se is an unlikely explanation for the findings here pertinent to either MA training effects or the moderating effects of level of education on MA training outcomes. As participants volunteered to take part in this 6-week long training program, to the extent that they were likely already cognitively engaged (see Stine-Morrow, 2007) this may explain the attenuation of effects particular to cognitive self-efficacy here (see Lachman et al., 1982).

\section{Concluding Remarks Regarding MA Training}

It may be that the effects we did find here could be seen in an even more positive light, in that the MA program (both in terms of the original treatmentcontrol comparisons and the replication of treatment effects among waiting list control participants when subsequently provided with MA training) did indeed have a measurable effect on this already high-functioning group speaks to its potency as an intervention. Likewise, the fact that $75 \%$ of the sample was willing to be reevaluated nearly a year later reinforces the fact that the program was perceived as efficacious to the extent that participants allowed themselves to be reinterviewed. Assuming that participants here had a history of seeking out cognitively stimulating activities and likely already 
felt a degree of cognitive self-efficacy prior to training, it could be inferred that the 6-week MA program must have offered them something unique in allowing them to more fully evaluate their abilities, interact with others creatively in a positive and process-oriented manner, and improve their affective health as well as their views about their everyday task vitality.

With this in mind, future intervention work with older adults should strive to access persons who are diverse educationally and regarding health to more fully explore the moderating roles of these factors on treatment outcomes, and extending the barometers of treatment efficacy to include everyday behavioral and affective outcomes. In this manner, interventions designed to enhance the cognitive skills of older persons whose focus is purportedly on cognitive functioning may also serve to improve affective functioning, improve physical health, enhance positive and stimulating interactions with others, and, indeed, improve persons' quality of life in later adulthood.

\section{Declaration of Conflicting Interests}

The authors declared no potential conflicts of interest with respect to the research, authorship, and/or publication of this article.

\section{Funding}

The authors received no financial support for the research, authorship, and/or publication of this article.

\section{References}

Albert, M. S., Jones, K., Savage, C. R., Berkman, L., Seeman, T., Blazer, D., \& Rowe, J. W. (1995). Predictors of cognitive change in older persons: MacArthur studies of successful aging. Psychology and Aging, 10, 578-589.

Andel, R., Crowe, M., Pedersen, N. L., Mortimer, J., Crimmins, E., Johansson, B., \& Gatz, M. (2005). Complexity of work and risk of Alzheimer's disease: A population-based study of aging and Swedish twins. Journal of Gerontology: Psychological Sciences, 60B, P251-P258.

Baltes, P. B., \& Willis, S. L. (1982). Plasticity and enhancement of intellectual functioning in old age: Penn State's Adult Development and Enrichment Program (ADEPT). In F. Craik \& S. Trehub (Eds.), Aging and cognitive processes (pp. 353-389). New York, NY: Plenum.

Blazer, D. (2002). Depression in later life. New York, NY: Springer.

Boron, J. B., Willis, S. L., \& Schaie, K. W. (2007). Cognitive training gain as a predictor of mental status. Journals of Gerontology: Psychological Sciences, 62, P45-P52.

Broadbent, D., Cooper, P., Fitzgerald, P., \& Parkes, K. (1982). The Cognitive Failures Questionnaire (CFQ) and its correlates. British Journal of Clinical Psychology, 21, 1-16. 
Cohen, J. (1988). Statistical power analysis for the behavioral sciences. Hillsdale, NJ: Prentice Hall.

Fried, L. P., Carlson, M. C., Freedman, M., Frick, K. D., Glass, T. A., Hill, J., . . Zeger, S. (2003). A social model for health, promotion in an aging population: Initial evidence on the Experience Corps model. Journal of Urban Health, 81, 64-78.

Hayslip, B. (1988). Personality-ability relationships in aged adults. Journal of Gerontology, 43, 79-84.

Hayslip, B. (1989a). Alternative mechanisms for improvements in fluid ability among aged persons. Psychology and Aging, 4, 122-124.

Hayslip, B. (1989b). Fluid ability training with aged people: A past with a future? Educational Gerontology, 15, 573-596.

Hayslip, B., \& Cooper, A. (2012). Subjective and objective intellectual change in older adults. Educational Gerontology, 38, 190-200.

Hayslip, B., \& Maiden, R. (2005). Cognitive loss. In C. N. Dulmus \& L. Rapp-Paglicci (Eds.), Handbook of prevention interventions for adults (pp. 27-55). New York, NY: John Wiley.

Hayslip, B., Paggi, K, Poole, M., \& Ward Pinson, M. (2009). The impact of mental aerobics training on memory impaired older adults. Clinical Gerontologist, 32, 389-394.

Hayslip, B., Servaty, H., \& Ward, A. (1996, April). Perceptions of everyday and psychometric intelligence and ability performance among older adults. Paper presented at the Biannual Cognitive Aging Conference, Atlanta, GA.

Hayslip, B., Servaty, H., Ward, A., \& Blackburn, J. (1995, November). Toward a definition of everyday cognition in later life. Paper presented at the annual scientific meeting of the Gerontological Society of America, Los Angeles, CA.

Hayslip, B., \& Sterns, H. (1979). Age differences in relationships between crystallized and fluid intelligences and problem solving. Journal of Gerontology, 34, 404-414.

Hayslip, B., \& Thomas, P. (1999, August). Stability of perceptions of intelligence among older adults. Paper presented at the annual convention of the American Psychological Association, Boston, MA.

Hertzog, C., Kramer, A. F., Wilson, R. S., \& Lindenberger, U. (2009). Enrichment effects on adult cognitive development: Can the functional capacity of older adults be preserved and enhanced? Psychological Science in the Public Interest, 9, 1-65.

Hess, T. M., Auman, C., Colcombe, S. J., \& Rahhal, T. A. (2003). The impact of stereotype threat on age differences in memory performance. Journal of Gerontology: Psychological Sciences, 58B, P3-P11.

Horn, J. L., \& Cattell, R. B. (1966). Refinement and test of the theory of fluid and crystallized general intelligence. Journal of Educational Psychology, 53, 253-270.

Horn, J. L., \& Cattell, R. B. (1967). Age differences in fluid and crystallized intelligence. Acta Psychologica, 26, 107-129.

Horn, J. L., \& Hofer, S. (1992). Major abilities and development during the adult period. In R. Sternberg \& C. Berg (Eds.), Intellectual development (pp. 44-99). New York, NY: Cambridge University Press. 
Hultsch, D. E., Hertzog, C., Small, B. J., \& Dixon, R. A. (1999). Use it or lose it: Engaged lifestyle as a buffer of cognitive decline in aging? Psychology and Aging, 14, 245-265.

Kooken, R., \& Hayslip, B. (1984). The use of stress inoculation in the treatment of test anxiety in older students. Educational Gerontology, 11, 39-58.

Lachman, M. E., Baltes, P. B., Nesselroade, J., \& Willis, S. (1982). Examination of personality-ability relationships in the elderly: The role of the contextual (interface) assessment mode. Journal of Research in Personality, 16, 485-501.

Paggi, K., \& Hayslip, B. (1999). Mental aerobics: Exercises for the mind in later life. Educational Gerontology, 25, 1-25.

Parisi, J. M., Greene, J. C., Morrow, D. G., \& Stine-Morrow, E. A. (2007). The senior odyssey: Participant experiences of a program of social and intellectual engagement. Activities, Adaptation \& Aging, 31, 31-49.

Park, D., Gutchess, A., Meade, M., \& Stine-Morrow, E. (2007). Improving cognitive function in older adults: Nontraditional approaches. Journals of Gerontology: Psychological Sciences and Social Sciences, 62B, 45-52.

Pashler, H., McDaniel, M., Roher, D., \& Bjork, R. (2009). Learning styles: Concepts and evidence. Psychological Science in the Public Interest, 9, 105-119.

Schaie, K. W., Willis, S. L., \& O'Hanlon, A. M. (1994). Perceived intellectual performance change over seven years. Journal of Gerontology: Psychological Sciences, 49, P108-P118.

Schooler, C., Mulatu, M., \& Oates, G. (1999). The continuing effects of substantively complex work of intellectual functioning. Psychology and Aging, 14, 483-505.

Sheikh, J. I., \& Yesavage, J. A. (1986). Geriatric Depression Scale (GDS): Recent evidence and development of a shorter version. In T. L. Brink (Ed.), Clinical gerontology: A guide to assessment and intervention (pp. 165-173). New York, NY: Haworth Press.

Snowdon, D. (2001). Aging with grace: What the Nun study teaches us about leading longer, healthier, and more meaningful lives. New York, NY: Bantam.

Stine-Morrow, E. A. L. (2007). The Dumbledore hypothesis of cognitive aging. Current Directions in Psychological Science, 16, 289-293.

Stine-Morrow, E. A. L., \& Basak, C. (2011). Cognitive interventions. In K. W. Schaie \& S. L. Willis (Eds.), Handbook of the psychology of aging (pp. 153-171). San Diego, CA: Academic Press.

Stine-Morrow, E. A. L., Parisi, J. M., Morrow, D. G., Greene, J. C., \& Park, D. C. (2007). An engagement model of cognitive optimization through adulthood. Journals of Gerontology: Psychological Sciences, 62, P62-P69.

Stine-Morrow, E. A. L., Parisi, J. M., Park, D. C., \& Morrow, D. G. (2008). The effects of an engaged lifestyle on cognitive vitality: A field experiment. Psychology and Aging, 23, 778-786.

Ware, J. E., Snow, K. K., Kosinski, M., \& Gandek, B. (1993). SF-36 health survey: Manual and interpretation guide. Boston, MA: The Health Institute, New England Medical Center. 
Williams, J., Plassman, B. L., Burke, J., Holsinger, T., \& Benjamin, S. (2010). Preventing Alzheimer's disease and cognitive decline (Evidence Report/ Technology Assessment Number 193). Rockville, MD: Agency for Healthcare Research and Quality, DHHS.

\section{Author Biographies}

Bert Hayslip Jr. is Regents Professor Emeritus at the University of North Texas. He is a fellow of the American Psychological Association, the Gerontological Society of America, and the Association for Gerontology in Higher Education. An associate editor of Experimental Aging Research and of Developmental Psychology, his coauthored books include Emerging Perspectives on Resilience in Adulthood and Later Life (Springer, 2012), Resilient Grandparent Caregivers: A Strengths-Based Perspective (Routledge, 2012), Adult Development and Aging (Krieger, 2011), and Parenting the Custodial Grandchild (Springer, 2008). He is Co-Principal Investigator (Co-PI) on a National Institute of Nursing Research (NINR)-funded project exploring interventions to improve the functioning of grandparent caregivers.

Kay Paggi received her Masters degree in Counselor Education from the University of North Texas. She has maintained an active and involved private practice as a Geriatric Care Manager and eldercare consultant in the Dallas-Ft.Worth Metroplex for over 20 years. In addition, she is highly involved in the community in presenting Mental Aerobics classes to older adults in the DFW area as well as being in demand in presenting many workshops on caregiving.

Daniela Caballero received her Masters degree in Clinical Psychology from the University of North Texas (UNT) and is currently enrolled in the doctoral program in Clinical Psychology at UNT. Her interests, which involve working with older adults as well as the intersection of culture and human development, enabled her to take an Clinical Internship at the Waco, TexasVeterans Affairs Hospital. 